\title{
Postnatal Cytomegalovirus Exposure in Infants of Antiretroviral-Treated and Untreated HIV-Infected Mothers
}

\author{
Sarah A. Meyer, ${ }^{1}$ Daniel J. Westreich, ${ }^{2}$ Emily Patel, ${ }^{3}$ Elizabeth P. Ehlinger, ${ }^{4}$ Linda Kalilani, ${ }^{5}$ \\ Rachel V. Lovingood, ${ }^{6}$ Thomas N. Denny, ${ }^{6}$ Geeta K. Swamy, ${ }^{3}$ and Sallie R. Permar ${ }^{6}$ \\ ${ }^{1}$ Department of Medicine, Children's Hospital Boston, Boston, MA 02115, USA \\ ${ }^{2}$ Department of Epidemiology, UNC-Chapel Hill, Chapel Hill, NC 27599, USA \\ ${ }^{3}$ Department of Obstetrics and Gynecology, Duke University Medical Center, Durham, NC 27710, USA \\ ${ }^{4}$ Division of Viral Pathogenesis, Beth Israel Deaconess Medical Center, Boston, MA 02115, USA \\ ${ }^{5}$ College of Medicine, University of Malawi, Blantyre, Malawi \\ ${ }^{6}$ Human Vaccine Institute, Duke University Medical Center, Durham, NC 27710, USA
}

Correspondence should be addressed to Sallie R. Permar; sallie.permar@duke.edu

Received 14 October 2013; Revised 3 January 2014; Accepted 23 January 2014; Published 3 March 2014

Academic Editor: Ann Duerr

Copyright (C) 2014 Sarah A. Meyer et al. This is an open access article distributed under the Creative Commons Attribution License, which permits unrestricted use, distribution, and reproduction in any medium, provided the original work is properly cited.

\begin{abstract}
HIV-1 and CMV are important pathogens transmitted via breastfeeding. Furthermore, perinatal CMV transmission may impact growth and disease progression in HIV-exposed infants. Although maternal antiretroviral therapy reduces milk HIV-1 RNA load and postnatal transmission, its impact on milk CMV load is unclear. We examined the relationship between milk CMV and HIV-1 load (4-6 weeks postpartum) and the impact of antiretroviral treatment in 69 HIV-infected, lactating Malawian women and assessed the relationship between milk CMV load and postnatal growth in HIV-exposed, breastfed infants through six months of age. Despite an association between milk HIV-1 RNA and CMV DNA load ( $0.39 \log _{10}$ rise CMV $\operatorname{load}_{\text {per }} \log _{10}$ rise HIV-1 RNA load, 95\% CI 0.13-0.66), milk CMV load was similar in antiretroviral-treated and untreated women. Higher milk CMV load was associated with lower length-for-age $(-0.53,95 \% \mathrm{CI}:-0.96,-0.10)$ and weight-for-age $(-0.40,95 \% \mathrm{CI}:-0.67,-0.13) Z$ score at six months in exposed, uninfected infants. As the impact of maternal antiretroviral therapy on the magnitude of postnatal CMV exposure may be limited, our findings of an inverse relationship between infant growth and milk CMV load highlight the importance of defining the role of perinatal CMV exposure on growth faltering of HIV-exposed infants.
\end{abstract}

\section{Introduction}

Despite significant scale-up of antiretroviral prophylaxis, 260,000 pediatric HIV-1 infections are still diagnosed annually $[1,2]$, of which nearly half are a result of breastfeeding [3]. In the United States, it is recommended that HIVinfected mothers use replacement feeding. However, replacement feeding in resource-poor countries is associated with increased mortality from diarrheal disease, pneumonia, and other infectious diseases [4]. In these regions, the World Health Organization recommends that HIV-infected women breastfeed their infants in the setting of maternal and/or infant antiretroviral prophylaxis, which considerably reduces postnatal HIV-1 transmission [5-7].

Another significant viral pathogen that is vertically transmitted is cytomegalovirus (CMV). Similar to HIV-1, CMV can be transmitted in utero, peripartum, or via breastfeeding. Congenital transmission has a well-established association with fetal growth restriction, sensorineural hearing loss, and neurodevelopmental abnormalities $[8,9]$. Prior to the advent of highly active antiretroviral therapy (HAART), reported congenital CMV rates were high among HIV-exposed and HIV-infected infants [10-12]. However, studies of congenital CMV in the HAART era show lower rates of congenital CMV in HIV-exposed, uninfected infants [13]. In contrast to congenital CMV transmission, peri- or postpartum CMV transmission is typically asymptomatic in healthy full-term infants. However, peripartum CMV acquisition in HIVinfected infants is an important predictor of morbidity and mortality [14], leading to higher rates of HIV-1 progression. Mounting evidence suggests that growth and development of HIV-exposed, uninfected infants are also adversely affected 
by perinatal CMV acquisition [15]. Moreover, high rates of symptomatic perinatal CMV infection have been described in HIV-exposed infants, an effect that may be modulated by maternal HAART [16]. Perinatal CMV infection may contribute to the recognized growth impairment of HIVexposed, uninfected infants. Thus, it is important to establish the pathogenesis of perinatal CMV transmission in HIVexposed infants and the impact of maternal HAART on infant CMV exposure.

CMV shedding in milk of CMV-seropositive women is common, with postnatal CMV transmission rates up to $70 \%$ [17-20]. Breast milk CMV load may be independently associated with the risk of postnatal CMV transmission [21, 22], especially in HIV-1-infected women as a direct correlation between CMV and HIV-1 breast milk viral loads has been described in untreated HIV-infected women [23]. While maternal HAART can effectively reduce HIV-1 RNA load in breast milk, its effect on CMV shedding is not established. Moreover, it is unclear if maternal HAART can reduce perinatal CMV transmission and associated disease. Establishing the impact of maternal HAART on CMV exposure and the effects of CMV exposure on growth and development of HIVexposed infants is important to improving infant survival in regions of high HIV-1 prevalence. In this study, we aimed to determine the impact of maternal HAART on milk CMV shedding. Further, we evaluated the impact of milk CMV load on the growth of breastfed, HIV-exposed infants.

\section{Materials and Methods}

2.1. Participants and Specimens. Sixty-nine HIV-1-infected pregnant women testing positive by rapid antibody test were recruited from two rural health clinics outside Blantyre, Malawi, between 2009 and 2010 and consented for enrollment in this pilot study $[24,25]$. The study and informed consent were approved by the College of Medicine Research and Ethics Committee in Malawi and institutional review boards at each participating US institution. Maternal peripheral blood CD4+ T-cell count and plasma HIV-1 RNA viral load were performed during the third trimester and women with confirmed infection were enrolled at delivery. Maternal antiretroviral use was assessed at each follow-up visit (delivery, 4-6 weeks, 3 months, and 6 months); untreated mothers and all infants were administered single dose Nevirapine at delivery. All women were also screened for CMV IgG by ELISA using the third trimester blood sample (Trinity Biotech). Breast milk from right and left breasts was collected separately between four to six weeks postpartum. Mothers were counseled to exclusively breastfeed for the first six months and were provided with a peanut-based food supplement for their own nutrition for six months postpartum. Per national guidelines at the time, HIV-infected mothers were counseled at six months postpartum to choose to continue breastfeeding or rapid weaning if replacement feeding was a viable option.

Infants were tested for HIV-1 infection by blood DNA PCR at birth (for in utero infection), four to six weeks of age (for peripartum infection), and every three months of life until weaning (for postnatal infection). Positive results were confirmed by repeated blood DNA PCR and plasma RNA load. Infant growth parameters of height (crown-heel length) and weight (by infant scale) were monitored at birth, four to six weeks, three months, and six months. The weightfor-length, length-for-age, and weight-for-age $Z$-scores were determined using the WHO Anthro program (v3.2.2). Congenital CMV infection was diagnosed by cord blood tested for CMV DNA PCR and IgM, as infant urine and saliva were not collected. No infants were positive for CMV IgM (Trinity Biotech). Thus, infants with positive CMV DNA PCR of cord blood were considered to be congenitally infected with CMV. All infants included in the growth analysis at six months of age were still breastfeeding.

2.2. Laboratory Assays. HIV-1 RNA load was measured using the Roche Cobas Ampliprep/Cobas TaqMan 48 for HIV1 load assay. Breast milk supernatant was diluted 1:5 in phosphate-buffered saline (PBS) prior to analysis, whereas plasma was diluted $1: 10$. The minimum levels of detection for this assay were 480 viral RNA copies/mL of plasma and 240 viral RNA copies/mL of breast milk. The laboratory performing these assays was enrolled in the National Institute of Allergy and Infectious Diseases Division of AIDS Virology Quality Assessment Program and certified for HIV-1 load determinations [25]. Milk CMV DNA load was determined by quantitative PCR as previously described [26]. The limit of quantification was 87 copies/mL. If virus amplification was detected but was below the level of the minimum virus standard, a value of half the minimum of detection was assigned. Sodium and potassium concentration of milk supernatant was measured using the Gen2 Ion Selective Electrode on the Roche Cobias c501 platform (Roche Diagnostics), with a sodium-potassium ratio $>1$ indicative of subclinical mastitis $[27,28]$.

2.3. Statistical Analysis. Distributions of demographic and laboratory characteristics were described using medians and interquartile ranges; differences between medians were described using two-sided $P$ values from Wilcoxon rank sum tests. We estimated the effects of HAART on CMV breast milk load using linear regression (crude and adjusted) for infant birth weight and gestational age and maternal mastitis, age, and CD4 count. Confidence intervals around the CMV and HIV-1 transmission rates were calculated with a continuity correction. For the analysis of viral load data in each breast, we applied a goodness-of-fit analysis (quasi-Akaike's information criterion) to determine whether an independent or exchange structure regression model was a better fit, and the results determined that the independent structure was a better fit. Thus, a generalized estimating equation (GEE) with an independent correlation matrix to control for the withinwoman correlation was used for the analysis of both breasts and each breast separately (right or left) from each individual woman. Cross-sectional associations between HIV-1 and CMV loads were described using scatterplots and linear regression to obtain slopes and $R^{2}$ values. Comparisons of the frequency of HIV-1 or CMV transmission between the 
groups were performed using the chi squared test. Finally, we estimated the effect of milk CMV DNA and HIV-1 RNA load at four to six weeks on infant growth $Z$-scores and the change in $Z$-scores for weight-for-length, length-for-age, and weightfor-age using linear regression both crude and adjusted for infant birth weight and gestational age and maternal CD4 count, age, and HAART use.

\section{Results}

3.1. Clinical Characteristics of HIV-Infected, CMV-Seropositive Lactating Women and Their Infants. Sixteen women initiated HAART (Trioimmune: Stavudine, Lamivudine, and Nevirapine) prior to enrollment, with two on therapy prior to pregnancy and 14 initiating HAART during pregnancy. Single dose Nevirapine was provided during labor to the remaining 53 untreated women and all infants. HAART-treated women were significantly older than untreated women. All women were CMV IgG seropositive. HAART-treated women had a similar peripheral CD4+ T-cell count to that of the untreated women during the third trimester (Table 1). Maternal plasma HIV-1 load during the third trimester was significantly lower in HAART-treated versus untreated women with $\log _{10}$ plasma HIV-1 RNA load 2.4 versus 4.0, respectively. Breast milk HIV-1 RNA load was detectable at 4-6 weeks postpartum in at least one breast in two of 16 women on HAART treatment $(12.5 \%)$ versus $33 / 53(62.3 \%)$ untreated women ( $P=0.0005$ by chi squared test). Thus, the median milk HIV-1 RNA load measured was similar between the two groups, but the range of the milk virus load was significantly higher in the untreated women (Table 1). Despite counseling on benefits of exclusive breastfeeding, of the 67 women who reported the date of initiation of mixed feeding, 10 women reported initiation of mixed feeding prior to 5 months of life (14.9\%), 26 women reported initiating mixed feeding in the infant's sixth month of life (38.8\%), 24 reported initiating mixed feeding after six months of age (35.8\%), and seven women reported not initiating mixed feeding before weaning at six months of life (10.4\%). While symptoms or signs of mastitis (such as breast soreness, erythema, or induration) were not reported by any of the subjects, subclinical mastitis was detected at four to six weeks postpartum in a similar proportion of treated and untreated women (Table 1).

In utero or peripartum $\mathrm{HIV}-1$ transmission occurred exclusively in the untreated group $(13 \%, 95 \%$ CI $[0.06,0.26])$, despite the use of single dose Nevirapine. Moreover, postpartum HIV-1 transmission occurred only in the untreated group $(6 \%, 95 \%$ CI $[0.01,0.17])$. When these modes of HIV1 transmission were combined, there was a trend towards a higher rate of vertical HIV-1 transmission in the untreated group compared to the treated group $(P=0.06$ by chi square test). Three infants were found to be congenitally infected with CMV by a positive cord blood CMV PCR: two in the treated and one in the untreated group and none of these overlapped with the HIV-infected infants. Low birth weight $(<2500 \mathrm{~g})$ was more prevalent in the HAART-treated $(25 \%)$ than the untreated (2\%) group. Moreover, preterm birth ( $<37$ weeks gestation), assessed by Ballard score [29], trended towards a higher incidence in the HAART-treated versus untreated group (Table 1). There was no association between preterm birth and CMV infection in this cohort $(P=$ 0.82 by chi square test), yet with only three congenital CMV infections in this cohort, the power to detect associations is limited.

\subsection{Effect of Maternal HAART and Subclinical Mastitis on} Milk CMV DNA Load. CMV load was quantitated in milk collected from each breast at four to six weeks postpartum. We assessed the viral loads in milk collected from each breast due to known potential discordance of HIV-1 RNA load between breasts and potential for unilateral mastitis $[25,28]$. The average CMV load from right and left breasts of HAARTtreated women was similar to that of untreated women (Table 1). To further assess the effect of HAART on milk CMV load, we determined the difference in $\log _{10}$ copies $/ \mathrm{mL}$ milk CMV load from both breasts and right and left breasts separately, associated with maternal HAART. There was no association between HAART and the magnitude of milk CMV load (Table 2). When the results were adjusted for maternal age, CD4 count, mastitis, and infant gestational age and birth weight, there was a trend towards a decrease in milk CMV DNA load in both breasts and right and left breasts with maternal HAART $\left(\log _{10}\right.$ difference in milk CMV DNA load: $-0.33,-0.21$, and -0.50 , resp.), though the $95 \%$ confidence intervals (CI) included zero in all comparisons.

We assessed the difference in $\log _{10}$ copies/mL milk CMV load from both breasts and right and left breasts, associated with subclinical mastitis. In both the raw and adjusted comparison of CMV load detected in milk, there was no association between mastitis and CMV load ( $\log _{10}$ difference in milk CMV load associated with subclinical mastitis: 0.23 , -0.47 , and 0.46 , for both breasts, left breast, and right breast, resp.). After adjustment for maternal age, HAART use, and CD4 count and infant birth weight and gestational age, the difference in milk CMV DNA load by subclinical mastitis remained nonsignificant. Thus, subclinical mastitis was not associated with elevated milk CMV load in our population.

Finally, we compared the milk CMV DNA load among HIV-transmitting and HIV-nontransmitting mothers, combining all HIV transmission modes in one group due to the small number of total HIV transmissions. Interestingly, the CMV DNA load was higher in transmitting mothers (mean $\log _{10}$ milk CMV DNA load $=4.99$ ) compared to nontransmitting mothers (mean $\log _{10}$ milk CMV DNA load $=3.81)(P=0.003)$. However, CMV DNA load in milk of mothers of congenitally CMV-infected infants (mean $\log _{10}$ milk CMV DNA load $=3.42$ ) was similar to that of mothers of infants who did not congenitally transmit CMV to their infants $\left(\right.$ mean $\log _{10}$ milk CMV DNA load $\left.=3.99\right)(P=0.31)$.

3.3. Relationship between HIV-1 RNA and CMV DNA Loads in Breast Milk. CMV DNA load in mucosal secretions has previously been directly correlated with the magnitude of the HIV-1 RNA load in the same compartment $[23,30]$. Thus, we assessed the cross-sectional relationship between milk HIV-1 RNA and CMV DNA load in our cohort of HAART-treated 
TABLE 1: Clinical characteristics of HIV-infected lactating mothers and their infants, comparing HAART-treated and untreated women.

\begin{tabular}{|c|c|c|c|}
\hline & Untreated $n=53$ & Treated $n=16$ & $P$ value \\
\hline \multicolumn{4}{|l|}{ Maternal characteristics } \\
\hline Age, years & $26(23,30)$ & $33(29,36)$ & 0.0002 \\
\hline Peripheral CD 4 count $(\text { cells } / \mu \mathrm{L})^{\mathrm{a}}$ & $382(217,494)$ & $315(257,481)$ & 0.74 \\
\hline Subclinical mastitis (milk K/Na $>1)^{\mathrm{b}}$ & $15(28 \%)$ & $4(25 \%)$ & 0.80 \\
\hline Plasma HIV-1 RNA load $\left(\log _{10} \text { copies } / \mathrm{mL}\right)^{\mathrm{b}}$ & $4.0(3.5,4.8)$ & $2.4(2.4,2.4)$ & $<0.0001$ \\
\hline Milk HIV-1 RNA load $\left(\log _{10} \text { copies } / \mathrm{mL}\right)^{\mathrm{b}}$ & $2.1(2.1,3.0)$ & $2.1(2.1,2.1)$ & $<0.0001$ \\
\hline Milk CMV DNA load $\left(\log _{10} \text { copies } / \mathrm{mL}\right)^{\mathrm{b}}$ & $4.0(3.2,4.6)$ & $4.0(3.2,5.3)$ & 0.83 \\
\hline \multicolumn{4}{|l|}{ Infant characteristics } \\
\hline In utero or peripartum HIV-1 acquisition & $7(13 \%)$ & $0(0 \%)$ & 0.19 \\
\hline Postpartum HIV-1 acquisition & $3(6 \%)$ & $0(0 \%)$ & 1.00 \\
\hline Congenital CMV acquisition & $1(2 \%)$ & $2(13 \%)$ & 0.13 \\
\hline Low birth weight & $1(2 \%)$ & $4(25 \%)$ & 0.009 \\
\hline Ballard score $<37$ weeks & $12(23 \%)$ & $7(44 \%)$ & 0.12 \\
\hline
\end{tabular}

Results reported as median (IQR: interquartile range) or $N(\%)$. Two-sided $P$ values for maternal characteristics derived from Wilcoxon two-sample test, normal approximation, or two-sided $P$ values for infant characteristics from Fisher's exact test. Significant $P$ values $(P<0.05)$ are bolded.

${ }^{a}$ Measured in the 3 rd trimester of pregnancy.

${ }^{\mathrm{b}}$ Measured at four to six weeks postpartum.

TABLE 2: Effect of maternal HAART on milk CMV DNA load at four to six weeks postpartum, measured as difference in milk $\log _{10} \mathrm{CMV}$ DNA load associated with HAART use.

\begin{tabular}{lcc}
\hline & Crude & Adjusted $^{\mathrm{a}}$ \\
\hline Both breasts & $0.08(-0.59,0.74)$ & $-0.33(-0.95,0.30)$ \\
Left breast & $0.16(-0.50,0.82)$ & $-0.21(-0.90,0.49)$ \\
Right breast & $-0.00(-0.66,0.65)$ & $-0.50(-1.18,0.17)$ \\
\hline
\end{tabular}

${ }^{\mathrm{a}}$ Results reported as difference in milk $\log _{10}$ CMV DNA associated with HAART use, controlling for infant gestational age and birth weight and maternal age, mastitis, and $\mathrm{CD} 4$ count.

and untreated HIV-infected women at four to six weeks postpartum. Using a linear regression model, we determined the rise in $\log _{10} \mathrm{CMV}$ copies/mL associated with a one $\log _{10}$ rise in HIV-1 copies/mL (Figure 1). The difference in $\log _{10}$ milk CMV DNA load for every one $\log _{10}$ rise in milk HIV-1 RNA load was estimated at 0.39 (95\% CI: $0.13-0.66)$ for both breasts, 0.28 for the right breast (95\% CI: $-0.03-0.60)$, and 0.49 for the left breast (95\% CI: $0.20-0.78$ ). However, there was no direct correlation between the milk viral loads in this cohort (correlation coefficient $=0.298,0.214$, and 0.377 for both, left, and right breasts). Thus, there was a weak association between the magnitude of the milk HIV-1 RNA and CMV DNA load in this cohort of HIV-infected, lactating women.

3.4. Association between Maternal Milk CMV DNA Load and Postnatal Growth of HIV-Exposed, Breastfed Infants. We evaluated the association between the magnitude of postnatal CMV exposure in HIV-exposed, uninfected infants and postnatal growth. Three of the HIV-exposed infants had a positive cord blood CMV DNA PCR, for a congenital CMV transmission rate of $4 \%$, similar to previous reports for HIVexposed infants $[13,31]$. For this analysis of postnatal CMV exposure and infant growth, we removed infants that were congenitally or perinatally infected with HIV-1 $(n=10)$ and congenitally infected with CMV $(n=3)$. One uninfected infant died prior to 6 months of age $(n=1)$; thus the analysis was performed on a total of 55 infants. Infant plasma was not available for further CMV testing after birth to determine the incidence of postnatal CMV acquisition. As described in Table 3, there was a significant reduction in crude and adjusted length-for-age $Z$-score and the weightfor-age $Z$-score at six months of age per $\log _{10}$ increase in milk CMV DNA load, with adjusted analysis controlling for birth weight and gestational age and maternal CD4 count, age, and HAART use. As exposure to CMV via breastfeeding is a postnatal exposure, we next assessed the change in the $Z$ score of growth parameters between four to six weeks and six months of age in these HIV-1 and CMV-exposed infants. The milk CMV DNA load remained only marginally negatively associated with the change in length-for-age in the adjusted analysis (-0.30; 95\% CI: $-0.86,0.25)$.

As the milk CMV DNA and HIV-1 RNA loads were weakly positively associated in this cohort, we tested whether milk HIV-1 RNA virus load was a better predictor of infant growth at six months. None of the associations between HIV1 RNA load and infant growth parameter $Z$-scores or change in growth parameter $Z$-scores at six months of age were significant (Table 3). However, there was a positive, though somewhat imprecise, association between infant weight-forlength at six months of age and HIV-1 RNA load in crude and adjusted analysis (Table 3 ). Thus, the correlations between milk CMV load and infant growth trends are stronger and more consistent than the associations between milk HIV-1 load and infant growth.

\section{Discussion}

HAART during pregnancy and breastfeeding is now standard practice for prevention of perinatal HIV-1 transmission [32]. 


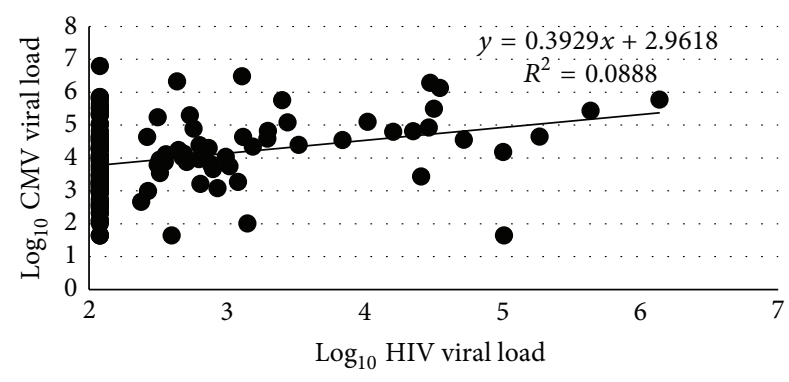

(a)

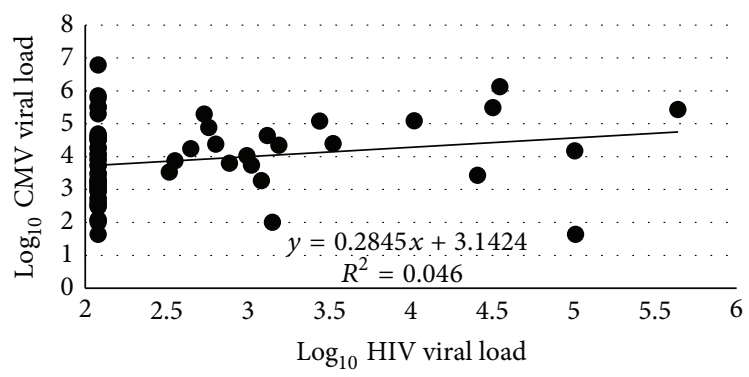

(b)

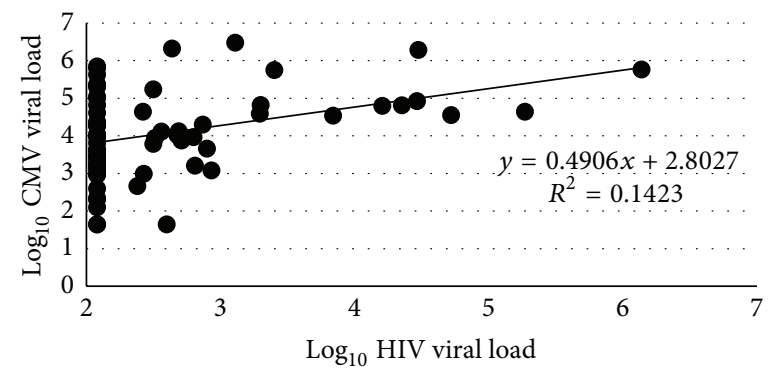

(c)
Linear change in $\log _{10}$ CMV DNA load for each $\log _{10}$ change in HIV RNA load $=0.39(0.13,0.66)$

Linear change in $\log _{10}$ CMV DNA load for each $\log _{10}$ change in HIV RNA load $=0.28(-0.03,0.60)$

Linear change in $\log _{10}$ CMV DNA load for each $\log _{10}$ change in HIV RNA load $=0.49(0.20,0.78)$

FIGURE 1: Cross-sectional associations between milk $\log _{10}$ HIV-1 RNA load and $\log _{10}$ CMV DNA load at four to six weeks postpartum, in both breasts (a), left breast only (b), and right breast only (c). Slope and $R^{2}$ values are indicated on each graph; the difference in log 10 CMV DNA load for each $\log _{10}$ difference in HIV-1 RNA load is presented to the right of each graph.

However, this study was initiated prior to establishment of the impact of maternal HAART on postnatal HIV-1 transmission, allowing us to compare breast milk CMV shedding and CMV transmission in HAART-treated and untreated mothers. In our cohort, HAART initiated prior to or during pregnancy successfully prevented in utero or postpartum HIV-1 transmission, further demonstrating the success of maternal antiretroviral prophylaxis. Interestingly, women on HAART were more likely to have a low-birthweight infant compared to untreated women (25\% versus $2 \%$, resp.), consistent with previous reports linking antiretrovirals during pregnancy with low birth weight [33-35]. However, this finding is in contrast to other recent data showing the lack of association between preterm birth and non-proteaseinhibitor-containing maternal HAART regimens [36-38]. This finding could also be linked to the older age of women on therapy compared to those that were not. To determine the impact of maternal HAART on infant perinatal CMV exposure, we focused on the relationship between HAART and milk CMV shedding.
Studies have associated perinatal CMV infection with increased morbidity and mortality, not only in HIVinfected infants but also in HIV-exposed, uninfected infants. These groups may have impaired fetal and infant growth attributable to perinatal CMV infection $[15,39]$. Despite the well-known risk of CMV and HIV-1 transmission via breast milk, breastfeeding is advocated as the primary source of infant nutrition in HIV-infected women in the developing world due to infeasible alternatives and high infant mortality associated with formula feeding [4]. Moreover, postnatal CMV transmission has been independently linked to breast milk CMV DNA load [21, 22]. Our study revealed a weak association in the magnitude of milk HIV-1 and CMV load, such that, for every HIV-1 $\log _{10}$ rise in HIV-1 load, there was a $0.39 \log _{10}$ increase in CMV load. Gianella et al. similarly demonstrated a correlation between CMV and HIV1 loads in other mucosal compartments, which is clinically significant as the presence of CMV is associated with HIV1 disease progression and mortality $[30,40]$. The correlation between CMV and HIV-1 shedding is independent of CD4+ 
TABLE 3: Association between breast milk $\log _{10}$ CMV DNA and HIV-1 RNA load and growth of breastfed HIV-exposed, uninfected infants.

\begin{tabular}{|c|c|c|c|c|}
\hline \multirow{2}{*}{ Outcome measure $(n=55)$} & \multicolumn{2}{|c|}{ CMV DNA load associations } & \multicolumn{2}{|c|}{ HIV-1 RNA load associations } \\
\hline & Crude & Adjusted $^{\mathrm{c}}$ & Crude & Adjusted \\
\hline Six-month $Z$-score ${ }^{\mathrm{a}}$ & $Z(95 \% \mathrm{CI})$ & $Z(95 \% \mathrm{CI})$ & $Z(95 \% \mathrm{CI})$ & $Z(95 \% \mathrm{CI})$ \\
\hline Weight for length & $\begin{array}{c}-0.05 \\
(-0.40,0.29)\end{array}$ & $\begin{array}{c}-0.03 \\
(-0.36,0.30)\end{array}$ & $\begin{array}{c}0.30 \\
(-0.03,0.63)\end{array}$ & $\begin{array}{c}0.40 \\
(-0.04,0.83)\end{array}$ \\
\hline Length for age & $\begin{array}{c}-0.48 \\
(-0.99,-0.08)\end{array}$ & $\begin{array}{c}-0.52 \\
(-0.93,-0.10)\end{array}$ & $\begin{array}{c}-0.17 \\
(-0.59,0.26)\end{array}$ & $\begin{array}{c}-0.35 \\
(-0.92,0.21)\end{array}$ \\
\hline Weight for age & $\begin{array}{c}-0.40 \\
(-0.67,-0.12)\end{array}$ & $\begin{array}{c}-0.39 \\
(-0.66,-0.13)\end{array}$ & $\begin{array}{c}0.13 \\
(-0.17,0.42)\end{array}$ & $\begin{array}{c}0.11 \\
(-0.26,0.47)\end{array}$ \\
\hline Change in $Z$-score ${ }^{b}$ & $\Delta Z(95 \% \mathrm{CI})$ & $\Delta Z(95 \% \mathrm{CI})$ & $\Delta Z(95 \% \mathrm{CI})$ & $\Delta Z(95 \% \mathrm{CI})$ \\
\hline Weight for length & $\begin{array}{c}0.63 \\
(-0.05,1.30)\end{array}$ & $\begin{array}{c}0.72 \\
(0.04,1.40)\end{array}$ & $\begin{array}{c}0.22 \\
(-0.47,0.91)\end{array}$ & $\begin{array}{c}-0.62 \\
(-1.50,0.27)\end{array}$ \\
\hline Length for age & $\begin{array}{c}-0.04 \\
(-0.56,0.48)\end{array}$ & $\begin{array}{c}-0.29 \\
(-0.84,0.26)\end{array}$ & $\begin{array}{c}-0.39 \\
(-0.93,0.15)\end{array}$ & $\begin{array}{c}0.20 \\
(-0.55,0.96)\end{array}$ \\
\hline Weight for age & $\begin{array}{c}0.20 \\
(-0.03,0.43)\end{array}$ & $\begin{array}{c}0.16 \\
(-0.05,0.38)\end{array}$ & $\begin{array}{c}-0.01 \\
(-0.25,0.23)\end{array}$ & $\begin{array}{c}-0.04 \\
(-0.34,0.26)\end{array}$ \\
\hline
\end{tabular}

${ }^{\mathrm{a}}$ Numbers in the table represent difference in $Z$-score at six months of age associated with a one-log ${ }_{10}$ increase in average breast milk CMV load.

${ }^{b}$ Numbers in the table represent difference in differences: the change in delta- $Z$-score between six months of age and four to six weeks of age associated with a one- $\log _{10}$ increase in average breast milk CMV load.

${ }^{c}$ Adjusted analysis accounts for infant birth weight and gestational age and maternal HAART use, age, and CD4 count.

T-cell count, plasma HIV-1 load, and other confounders $[23,41]$. Despite these associations of mucosal HIV-1 and CMV shedding, our analysis revealed only minimal impact of HAART on breast milk CMV load, suggesting that expanded maternal use of antiretroviral therapy may have a limited impact on infant postnatal CMV exposure.

HIV-exposed, uninfected infants have a growth disadvantage compared to their unexposed counterparts [15], though the underlying pathophysiology is not understood. As congenital CMV infection is independently associated with in utero growth restriction, it is reasonable to consider whether postnatal CMV exposure or infection plays a role in the growth outcome of HIV-exposed, uninfected infants [42]. We found a reduced length-for-age $Z$-score $(-0.53)$ and weightfor-age $Z$-score $(-0.40)$ for each $\log _{10}$ increase in milk CMV load at 6 months of age in HIV-exposed, uninfected infants. However, the CMV milk DNA load remained only marginally negatively associated with the change in length-for-age $Z$ score between one and six months. Thus, the negative association of milk CMV DNA load with infant growth may only be significant in the early period of breastfeeding or only reflect the magnitude of the peripartum CMV exposure. Mixed feeding rates were high in this infant cohort before 6 months of life (14.9\% prior to 5 months of age and $38.8 \%$ in the 6th month of life); thus, the growth rate over the first six months may have been impacted by this infant feeding pattern. Alternatively, high magnitude milk CMV shedding may be associated with maternal health status that is negatively impacting fetal and infant growth independently, as women that shed $\mathrm{CMV}$ at higher rates may be more immunocompromised and nutritionally deficient.

The limitations of this hypothesis-generating pilot study include a relatively small maternal sample size and lack of infant urine or saliva for confirmatory diagnosis of CMV congenital and postnatal CMV acquisition. Despite its small sample size, this study is unique in its enrollment of both HAART-treated and untreated mothers, as it enrolled prior to the establishment of maternal ARV treatment during breastfeeding as highly preventative against postnatal HIV transmission, elevating the importance of our analysis of CMV and HIV-1 load in milk and infant growth in this cohort. With these limitations in mind, we have demonstrated that maternal HAART does not have a large impact on breast milk CMV shedding, indicating that postnatal CMV exposure for HIV-exposed infants will continue at a similar level despite increasing maternal HIV-1 therapy/prophylaxis during breastfeeding. Moreover, the negative association between milk CMV load and postnatal growth in HIVexposed infants in this small study is intriguing and should be further assessed in larger clinical studies, determining whether reduction of postnatal CMV exposure is important to improving the developmental outcome of HIV-exposed infants. Additional larger studies of the kinetics of maternal CMV shedding, postnatal transmission, and infant growth in the setting of maternal HAART would now be important given the current standard of care. Further understanding of the interaction between CMV and HIV-1 may establish methods to reduce the morbidity and growth impairment of HIVexposed infants and maximize the benefits of breastfeeding in low-resource countries.

\section{Conflict of Interests}

The authors declare no conflict of interests.

\section{Authors' Contribution}

Geeta K. Swamy and Sallie R. Permar contributed equally to the work. 


\section{Acknowledgments}

This work was supported by the National Institute of Allergy and Infectious Diseases of the National Institutes of Health by the Center for HIV/AIDS Vaccine Immunology (U19AI067854), the Doris Duke Charitable Foundation (SRP), and the Children's Hospital Boston Fred Lovejoy Research Award (SAM). The authors thank Ebbie Chalaluka, Dr. Steve Meshnick, clinical staff, and study participants at the UNC Malaria Project in Blantyre, Malawi.

\section{References}

[1] UNAIDS, Report on the global AIDS epidemic 2010, http:// www.unaids.org/globalreport/Global_report.htm.

[2] UNAIDS, “2013 Report on the Global AIDS Epidemic," 2013.

[3] WHO, http://www.who.int/child_adolesce_health/documents/ 9789241596596/en/index.html.

[4] C. G. Victora and A. J. D. Barros, "Effect of breastfeeding on infant and child mortality due to infectious diseases in less developed countries: a pooled analysis," The Lancet, vol. 355, no. 9202, pp. 451-455, 2000.

[5] R. L. Shapiro, M. D. Hughes, A. Ogwu et al., "Antiretroviral regimens in pregnancy and breast-feeding in Botswana," The New England Journal of Medicine, vol. 362, no. 24, pp. 22822294, 2010.

[6] C. S. Chasela, M. G. Hudgens, D. J. Jamieson et al., "Maternal or infant antiretroviral drugs to reduce HIV-1 transmission," The New England Journal of Medicine, vol. 362, no. 24, pp. 2271-2281, 2010.

[7] N. I. Kumwenda, D. R. Hoover, L. M. Mofenson et al., "Extended antiretroviral prophylaxis to reduce breast-milk HIV-1 transmission," The New England Journal of Medicine, vol. 359, no. 2, pp. 119-129, 2008.

[8] A. Ornoy and O. Diav-Citrin, "Fetal effects of primary and secondary cytomegalovirus infection in pregnancy," Reproductive Toxicology, vol. 21, no. 4, pp. 399-409, 2006.

[9] R. F. Pass, K. B. Fowler, S. B. Boppana, W. J. Britt, and S. Stagno, "Congenital cytomegalovirus infection following first trimester maternal infection: symptoms at birth and outcome," Journal of Clinical Virology, vol. 35, no. 2, pp. 216-220, 2006.

[10] M. Doyle, J. T. Atkins, and I. R. Rivera-Matos, "Congenital cytomegalovirus infection in infants infected with human immunodeficiency virus type," Pediatric Infectious Disease Journal, vol. 15, no. 12, pp. 1102-1106, 1996.

[11] A. Kovacs, M. Schluchter, K. Easley et al., "Cytomegalovirus infection and HIV-1 disease progression in infants born to HIV1-infected women," The New England Journal of Medicine, vol. 341, no. 2, pp. 77-84, 1999.

[12] S. Chandwani, A. Kaul, D. Bebenroth et al., "Cytomegalovirus infection in human immunodeficiency virus type 1-infected children," Pediatric Infectious Disease Journal, vol. 15, no. 4, pp. 310-314, 1996.

[13] G. Guibert, J. Warszawski, J. L. Chenadec et al., "Decreased risk of congenital cytomegalovirus infection in children born to HIV-1-infected mothers in the era of highly active antiretroviral therapy," Clinical Infectious Diseases, vol. 48, no. 11, pp. 15161525, 2009.

[14] J. A. Slyker, B. L. Lohman-Payne, G. C. John-Stewart et al., "Acute cytomegalovirus infection in Kenyan HIV-infected infants," AIDS, vol. 23, no. 16, pp. 2173-2181, 2009.
[15] U. A. Gompels, N. Larke, M. Sanz-Ramos et al., "Human cytomegalovirus infant infection adversely affects growth and development in maternally HIV-exposed and unexposed infants in Zambia," Clinical Infectious Diseases, vol. 54, no. 3, pp. 434-442, 2012.

[16] T. Frederick, J. Homans, L. Spencer et al., "The effect of prenatal highly active antiretroviral therapy on the transmission of congenital and perinatal/early postnatal cytomegalovirus among HIV-infected and HIV-exposed infants," Clinical Infectious Diseases, vol. 55, no. 6, pp. 877-884, 2012.

[17] M. Dworsky, M. Yow, S. Stagno, R. F. Pass, and C. Alford, "Cytomegalovirus infection of breast milk and transmission in infancy," Pediatrics, vol. 72, no. 3, pp. 295-299, 1983.

[18] K. Hamprecht, J. Maschmann, M. Vochem, K. Dietz, C. P. Speer, and G. Jahn, "Epidemiology of transmission of cytomegalovirus from mother to preterm infant by breastfeeding," The Lancet, vol. 357, no. 9255, pp. 513-518, 2001.

[19] W.-T. Jim, C.-H. Shu, N.-C. Chiu et al., "Transmission of cytomegalovirus from mothers to preterm infants by breast milk," The Pediatric Infectious Disease Journal, vol. 23, no. 9, pp. 848-851, 2004.

[20] M. Vochem, K. Hamprecht, G. Jahn, and C. P. Speer, "Transmission of cytomegalovirus to preterm infants through breast milk," Pediatric Infectious Disease Journal, vol. 17, no. 1, pp. 5358, 1998.

[21] W.-T. Jim, C.-H. Shu, N.-C. Chiu et al., "High cytomegalovirus load and prolonged virus excretion in breast milk increase risk for viral acquisition by very low birth weight infants," Pediatric Infectious Disease Journal, vol. 28, no. 10, pp. 891-894, 2009.

[22] K. Hamprecht, J. Maschmann, G. Jahn, C. F. Poets, and R. Goelz, "Cytomegalovirus transmission to preterm infants during lactation," Journal of Clinical Virology, vol. 41, no. 3, pp. 198-205, 2008.

[23] S. Gantt, J. Carlsson, A. K. Shetty et al., "Cytomegalovirus and Epstein-Barr virus in breast milk are associated with HIV-1 shedding but not with mastitis," AIDS, vol. 22, no. 12, pp. 14531460, 2008.

[24] S. P. Meshnick, Pilot Study of Immunologic and Virologic Correlates of Maternal To Child HIV-1 Transmission via Breast Milk, Division of AIDS, U.S. National Institute of Allergy and Infectious Diseases U.S. National Institutes of Health, 2007.

[25] J. F. Salazar-Gonzalez, M. G. Salazar, G. H. Learn et al., “Origin and evolution of HIV-1 in breast milk determined by singlegenome amplification and sequencing," Journal of Virology, vol. 85, no. 6, pp. 2751-2763, 2011.

[26] E. P. Ehlinger, E. M. Webster, H. H. Kang et al., "Maternal cytomegalovirus-specific immune responses and symptomatic postnatal cytomegalovirus transmission in very low-birthweight preterm infants," The Journal of Infectious Diseases, vol. 204, no. 11, pp. 1672-1682, 2011.

[27] R. D. Semba, N. Kumwenda, T. E. Taha et al., "Mastitis and immunological factors in breast milk of lactating women in Malawi," Clinical and Diagnostic Laboratory Immunology, vol. 6, no. 5, pp. 671-674, 1999.

[28] J. F. Willumsen, S. M. Filteau, A. Coutsoudis, K. E. Uebel, M.-L. Newell, and A. M. Tomkins, "Subclinical mastitis as a risk factor for mother-infant HIV transmission," Advances in Experimental Medicine and Biology, vol. 478, pp. 211-223, 2000.

[29] J. L. Ballard, K. K. Novak, and M. Driver, "A simplified score for assessment of fetal maturation of newly born infants," Journal of Pediatrics, vol. 95, no. 5, pp. 769-774, 1979. 
[30] S. Gianella, C. M. Anderson, M. V. Vargas et al., "Cytomegalovirus DNA in semen and blood is associated with higher levels of proviral HIV DNA," The Journal of Infectious Diseases, vol. 207, no. 6, pp. 898-902, 2013.

[31] W. Khamduang, G. Jourdain, W. Sirirungsi et al., "The interrelated transmission of HIV-1 and cytomegalovirus during gestation and delivery in the offspring of HIV-infected mothers," Journal of Acquired Immune Deficiency Syndromes, vol. 58, no. 2, pp. 188-192, 2011.

[32] WHO, "Antiretroviral drugs for treating pregnant women and preventing HIV infection in infants," 2010.

[33] D. H. Watts, P. L. Williams, D. Kacanek et al., "Combination antiretroviral use and preterm birth," The Journal of Infectious Diseases, vol. 207, no. 4, pp. 612-621, 2013.

[34] J. Y. Chen, H. J. Ribaudo, S. Souda et al., "Highly active antiretroviral therapy and adverse birth outcomes among HIVinfected women in Botswana," The Journal of Infectious Diseases, vol. 206, no. 11, pp. 1695-1705, 2012.

[35] C. L. Townsend, J. Schulte, C. Thorne et al., "Antiretroviral therapy and preterm delivery-a pooled analysis of data from the United States and Europe," British Journal of Obstetrics and Gynaecology, vol. 117, no. 11, pp. 1399-1410, 2010.

[36] E. Azria, C. Moutafoff, T. Schmitz et al., "Pregnancy outcomes in women with HIV type-1 receiving a lopinavir/ritonavircontaining regimen," Antiviral Therapy, vol. 14, no. 3, pp. 423432, 2009.

[37] Kesho Bora Study Group and I. de Vincenzi, "Triple antiretroviral compared with zidovudine and single-dose nevirapine prophylaxis during pregnancy and breastfeeding for prevention of mother-to-child transmission of HIV-1 (Kesho Bora study): a randomised controlled trial," The Lancet Infectious Diseases, vol. 11, no. 3, pp. 171-180, 2011.

[38] K. Patel, D. E. Shapiro, S. B. Brogly et al., "Prenatal protease inhibitor use and risk of preterm birth among HIV-infected women initiating antiretroviral drugs during pregnancy," The Journal of Infectious Diseases, vol. 201, no. 7, pp. 1035-1044, 2010.

[39] J. A. Slyker, B. L. Lohman-Payne, S. L. Rowland-Jones et al., "The detection of cytomegalovirus DNA in maternal plasma is associated with mortality in HIV-1-infected women and their infants," AIDS, vol. 23, no. 1, pp. 117-124, 2009.

[40] C. M. Parrinello, E. Sinclair, A. L. Landay et al., "Cytomegalovirus immunoglobulin G antibody is associated with subclinical carotid artery disease among HIV-infected women," The Journal of Infectious Diseases, vol. 205, no. 12, pp. 1788-1796, 2012.

[41] S. M. Whitcup, E. Fortin, A. S. Lindblad et al., "Discontinuation of anticytomegalovirus therapy in patients with HIV infection and cytomegalovirus retinitis," The Journal of the American Medical Association, vol. 282, no. 17, pp. 1633-1637, 1999.

[42] Y. Maruyama, H. Sameshima, M. Kamitomo et al., "Fetal manifestations and poor outcomes of congenital cytomegalovirus infections: possible candidates for intrauterine antiviral treatments," Journal of Obstetrics and Gynaecology Research, vol. 33, no. 5, pp. 619-623, 2007. 


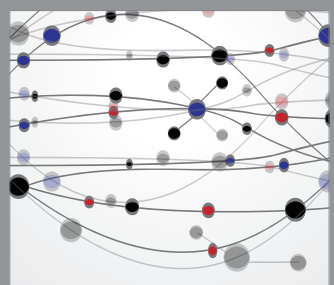

The Scientific World Journal
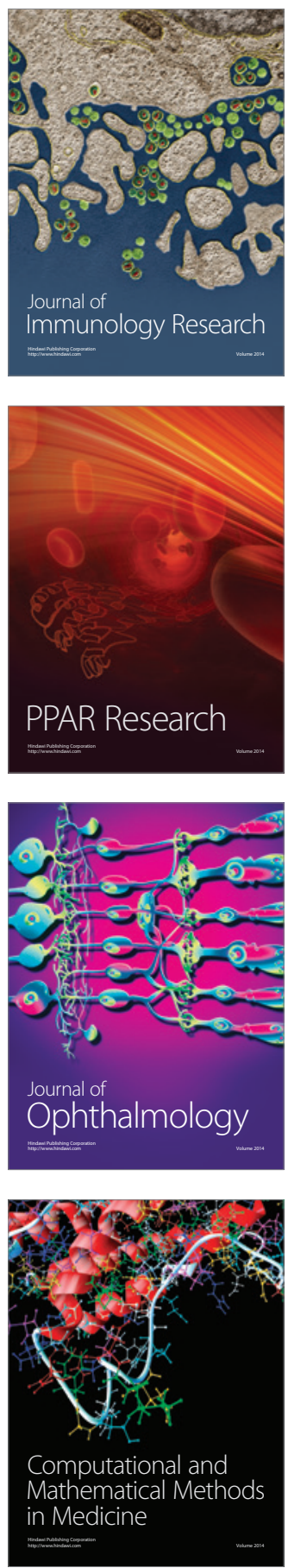

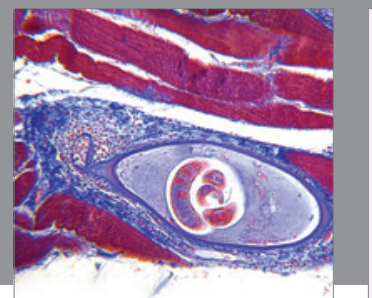

Gastroenterology

Research and Practice
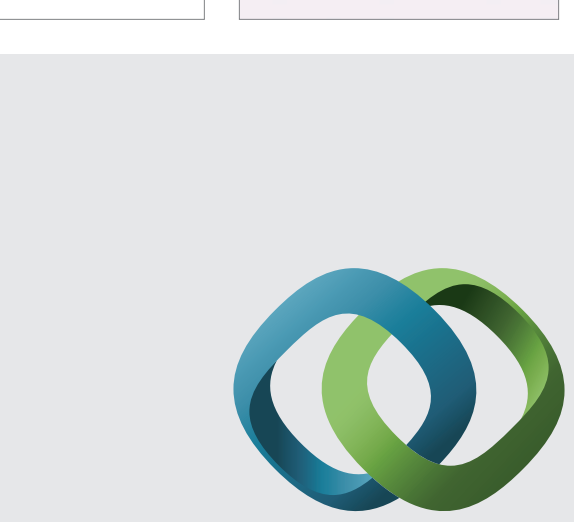

\section{Hindawi}

Submit your manuscripts at

http://www.hindawi.com
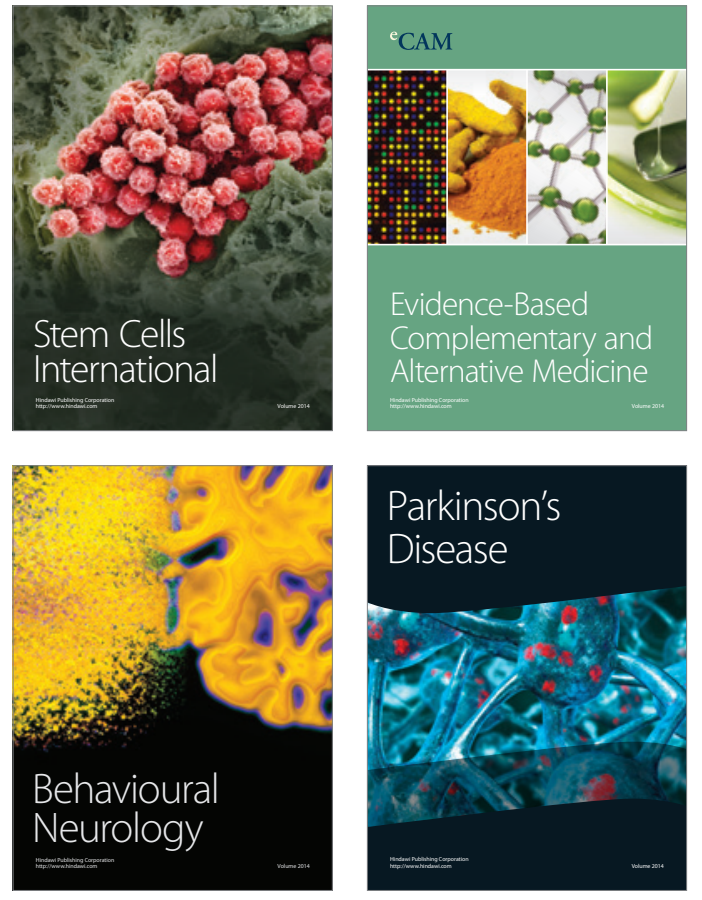
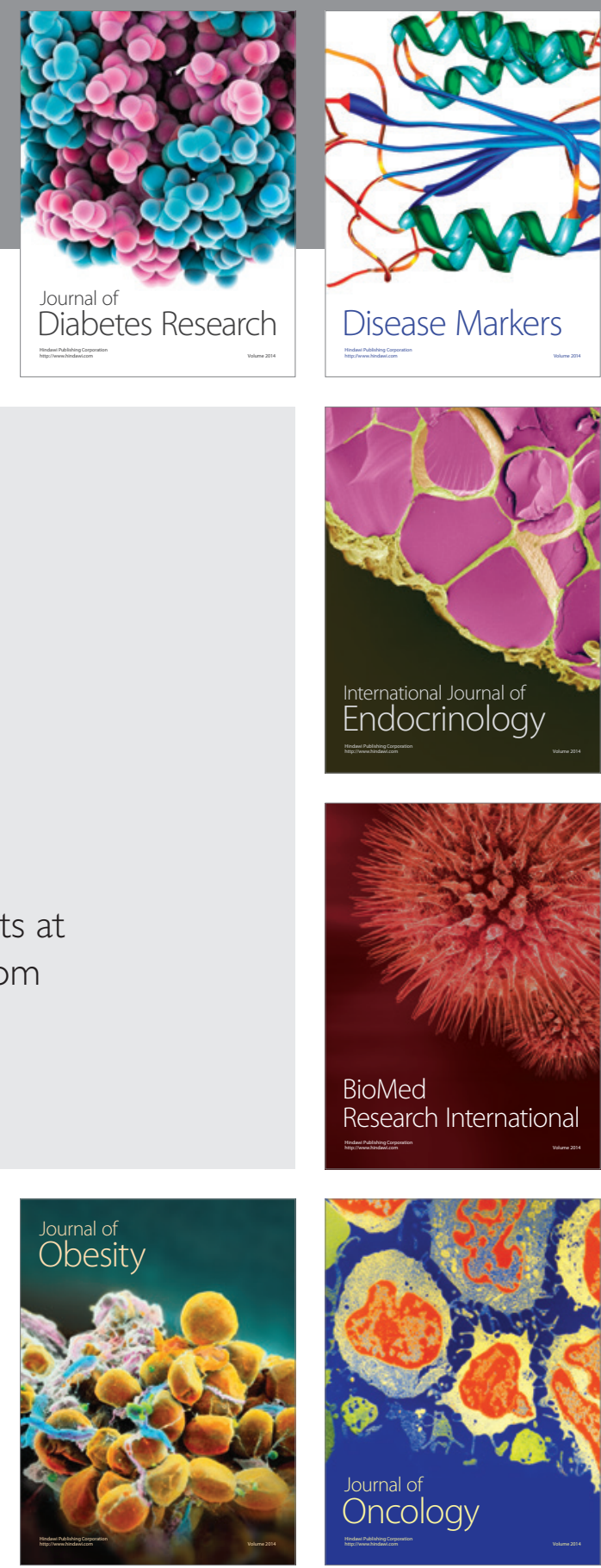

Disease Markers
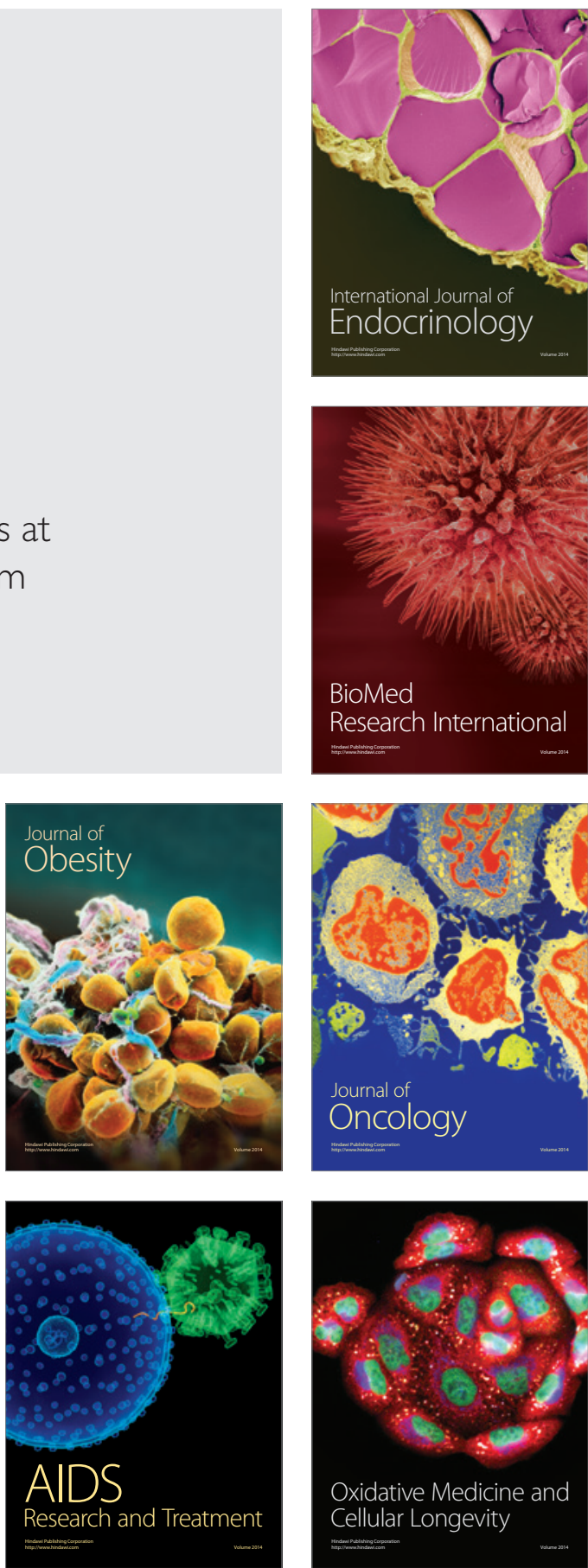